\title{
Challenges within our current education model and where to direct innovation: the three-strand triquetra approach
}

\author{
Sanjay P. Prabhu ${ }^{1}$ (D) $\cdot$ Micheál A. Breen ${ }^{1}$ \\ Received: 1 September 2020 / Revised: 1 September 2020 / Accepted: 9 November 2020 / Published online: 27 November 2020 \\ (C) Springer-Verlag GmbH Germany, part of Springer Nature 2020
}

\section{Introduction}

Traditional education in many pediatric radiology departments is based on an apprenticeship-type model, in which trainees primarily learn on the job, with graded levels of guidance and supervision from attending faculty members. More recently, this framework has come under threat in many pediatric radiology departments, where higher patient volumes, demands for greater speed and efficiency, increasing complexity of our patients, clinical questions and imaging studies, and management decisions have all resulted in increasing demands on the pediatric radiologist's time and energy. These factors are compounded at many institutions worldwide by a shortage of pediatric radiologists. We now know that this shortage results in increased burnout among pediatric radiologists and that this might be the most important challenge facing pediatric radiology education [1]. The emergence of the coronavirus disease 2019 (COVID-19) pandemic has exacerbated these challenges but has also offered opportunities for innovation and growth [2].

There are studies that support the perception of many academic radiologists that incorporating informal resident or fellow teaching into a clinical session leads to decreased clinical productivity [3]. Although trainees help clinical workflow by providing initial reviews of imaging studies, generating reports, protocolling studies and providing consults on reported studies to referring clinicians, this benefit is frequently offset by the time required of the attending pediatric radiologist to supervise and teach trainees, provide hands-on procedural training in US, fluoroscopy and intervention, and contact referring providers when there are substantive changes to

Sanjay P. Prabhu

sanjay.prabhu@childrens.harvard.edu

1 Department of Radiology, Boston Children's Hospital and Harvard Medical School, 300 Longwood Ave., Boston, MA 02115, USA preliminary trainee reports or recommendations. At our institution, most faculty members spend additional time after scheduled clinical sessions reviewing and editing trainees' reports. This lengthens the work day, encroaches on "protected" academic and personal time, and increases the risk of burnout. While there is increased emphasis on measuring and documenting clinical productivity in terms of average relative value units, it is not easy to track, document and recognize efforts directed toward non-interpretive tasks, particularly educational efforts toward radiology trainees, medical students, non-radiologist clinicians and support staff.

From the trainees' point of view, an increased number of studies per session means a greater focus on clinical productivity, often at the expense of the learning process. While some might argue that exposure to larger number of cases per session improves trainees' experience, there is a negative correlation between increased numbers of studies and number of discrepancies between trainee-generated reports and the final report [4]. Moreover, this issue has been recently complicated by the COVID-19 pandemic. The pandemic has resulted in decreased clinical volumes at academic pediatric centers, a move toward "remote/virtual" reading rooms to promote physical distancing, unique difficulties teaching trainees and allowing them to perform hands-on procedures including fluoroscopy, US and emergent procedures like intussusception reduction, and in some cases, reassignment of radiology residents to rotations other than their previously scheduled pediatric radiology rotations. A real concern of the pediatric radiology community in 2020 is that the pandemic will further limit our opportunities to mentor, guide and inspire residents toward a career as a pediatric radiologist, thereby compounding the problem of staff shortages and the secondary effects on education in our subspecialty. But we believe we can turn this historic challenge into an opportunity for educational innovation and growth with a three-strand "triquetra" approach (Fig. 1).

The first strand is focused on improving clinical efficiency by reducing time spent on repetitive or non-interpretive tasks 
Where to direct innovation in pediatric radiology education: the three-strand triquetra approach

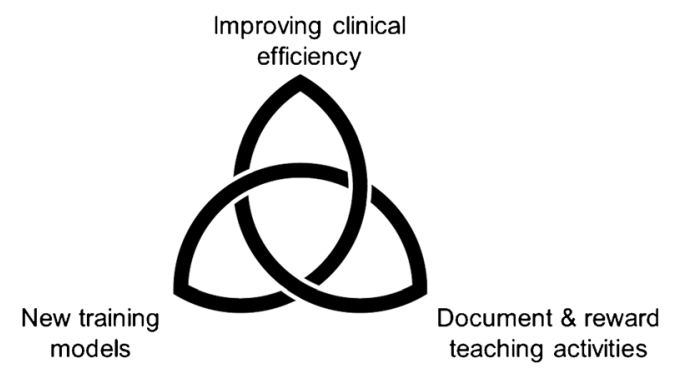

Fig. 1 Graphic demonstrates the three strands of the triquetra approach to pediatric radiology education

and using the attending radiologist's time saved or gained to invest in education during clinical sessions. The second strand deals with developing training models that re-imagine certain aspects of radiologist education content and delivery outside the reading room including online education and simulation, while retaining the time-proven apprenticeship model of reading room sign-out and hands-on training in the US/ fluoroscopy suite. The third strand is focused on ways to accurately track, measure and reward time spent by faculty on educational activities including informal activities that historically have not been objectively documented.

\section{Improving clinical efficiency}

Tools to improve clinical efficiency in radiology departments must be focused on eliminating unnecessary or repetitive tasks and reducing interruptions to workflow [5]. Some of these tools involve tweaks to the existing information-technology system like integrating the picture archiving and communication system, dictation and electronic medical record (EMR), thereby launching studies with a single-click automated data import from the EMR and imaging equipment into dictation templates along with standardized structured reporting and ready access to radiology decision support tools. Another tool that is readily accessible at most institutions is the radiologistcentric view of the EMR, which summarizes the patient's diagnostic odyssey, enabling a rapid understanding of the clinical problem in complex cases. Other innovations that can help radiologists shorten the time spent on tedious, timeconsuming tasks include developing and deploying artificial intelligence/machine learning applications like bone age reporting tools and automated tumor size measurements [6].

Other interventions to reduce time spent by radiologists on non-interpretive tasks include training non-physician reading room assistants/coordinators to contact clinical colleagues when more clinical information is required for a requested imaging study or to communicate important time-sensitive results to clinical colleagues. Automated systems have also been proved effective in this area [7]. At our institution, we have found that adding a dedicated consult radiologist to discuss complex cases with clinical teams allows other radiologists to focus on patient care and trainee education; this is a role that can be shared by trainees and faculty.

\section{New training models}

The traditional didactic lecture and case conference remained the mainstay of teaching in most radiology residency programs and pediatric radiology fellowships until the emergence of the COVID-19 pandemic early this year; since then, there has been a rapid shift toward online teaching conferences and webinars [2]. However, the format of most online lectures remains similar to that of didactic lectures or the case conference, with static images and bullet points on slides describing the imaging findings of a particular condition. What has changed is the ability of trainees to rapidly access a huge number of complementary or competing education resources using the smartphone that seems omnipresent in their hands. This trend had already sparked much debate pre-COVID, with some faculty members complaining that the practice seemed disrespectful from the vantage point of the lectern. But have we not all grown accustomed to searching online for real-time information about disease entities, imaging protocols and differential diagnoses at the workstation?

Innovation in pediatric radiology education cannot ignore that teacher and learner now have access to the World Wide Web in the palm of their hand. At the same time, we should remain mindful of the negative effects such technology can have on learners' attention and memory systems. There is some evidence that heavy use of modern technology and social media in education can endanger the capacity to think and remember clearly, decrease concentration spans and imperil creativity. The tendency to get distracted increases further with online lectures than in-person lectures. Studies have shown that maximum median engagement for a video of any length is around $6 \mathrm{~min}$ and expecting unsupervised trainees to watch video lectures longer than $10 \mathrm{~min}$ is likely to be wasted effort [8].

With this background, it is clear that although technology is revolutionizing how trainees learn, it is important to explore ways to make the case conference and didactic lectures more interactive and increase audience participation. Such techniques include flipped classrooms and workstation simulation using online case repositories that trainees can actively report and learn from with expert guidance from a faculty member. These can be supplemented with radiologic-pathological correlation of atypical presentations of common cases that might not be available in an online resource and can be used to engage the audience and ensure deeper understanding of the material.

Other techniques to actively engage trainees include audience response or gamification of the case conference, assigning 
groups of trainees to work on separate parts of a topic to teach their peers, and weekly conferences where residents summarize the pearls and pitfalls they have encountered during their week at the workstation. The emphasis in these scenarios is on creating an active learning environment, setting realistic expectations of trainees and, most important, measuring trainees' understanding of a topic at the end of the exercise. For example, success of the flipped classroom depends on creation of bitesize video or text content with clear objectives for the session, engagement in the classroom reinforcing the key concepts emphasized in the pre-class content, and measurement of understanding using active learning exercises [9].

As an extension of the online lecture, recordings of lectures on both basic and advanced topics can be created by academic institutions. These repositories of curated lectures can then form the basis of virtual curriculum for self-directed learning. Such a curriculum can be supplemented at the institutional level with flipped classrooms, workstation simulations and case conferences, as mentioned in the preceding paragraph, but with so many competing distractions for the $21^{\text {st }}$-century learner, we also believe that collaboration at the societal level with professional organizations like the Society for Pediatric Radiology can play an important role in curating and delivering a core curriculum for all.

Another avenue that has not been fully explored is the use of simulation for development of hands-on skills like pediatric US and fluoroscopic examinations. In small studies, trainees expressed greater confidence when faced with real-life scenarios after learning a critical skill like cranial US on a realistic simulated model [10]. Such tools might also help mitigate the shortage of opportunities for trainees to reduce intussusceptions or perform procedures like lumbar puncture or renal biopsies [11].

\section{Documenting and rewarding teaching activities}

Although technology is changing the way the current generation of radiology trainees learn, there remain distinct advantages to in-person education and training. Trainees continue to require interaction with teachers and role models; neither competence nor excellence in pediatric radiology can be obtained from gadgets and social media alone. Creating the right environment for in-person teaching to occur is extremely important in our specialty, which requires adapting factual knowledge to real-life situations and hands-on procedures.

The time and effort attending radiologists spend on this aspect of developing our specialty is crucial for engaging medical students and radiology residents and for training pediatric radiology fellows and junior faculty. This work must be documented and incorporated into the productivity graph of academic radiologists. Regular assessments of faculty members by trainees should be the norm, not the exception. This recognition of the teaching efforts will encourage academic pediatric radiologists to dedicate time to this important activity and move our subspecialty forward by developing thoughtful and well-trained young pediatric radiologists. Documenting educational activities outside clinical sessions such as lectures given to medical students, radiology trainees and others should be standardized.

As more radiology education becomes virtual, it is important to recognize the time and upfront effort required to adapt to new technology and delivery platforms and convert traditional lectures to incorporate newer teaching techniques like the flipped classroom. These efforts should be recognized, not only for the early adopters who face the challenges of doing this with minimal resources, but also the later entrants who are finding their conversion expedited by current circumstances. As education migrates toward newer platforms like social media, we need to train and reward such contributions if we are to thrive as a $21^{\text {st }}$-century subspecialty.

\section{Conclusion}

There are certainly many challenges to our current education model in pediatric radiology. No other subspecialty requires the radiologist to be knowledgeable in as many different body systems, among as diverse a patient population and in as many different imaging modalities, not to mention the interpersonal and noninterpretive skills required to be an effective team member in the unique setting of pediatric medicine. Such challenges have always existed and always will. As a subspecialty, we have historically shown a huge ability to innovate and adapt, and we should view the challenges we face now as an opportunity to use technology and human resources to enable our educational mission and improve the outcomes for our young patients. We have to ensure that the willingness and enthusiasm of our faculty and trainees to face these challenges is matched with appropriate education, time and resources. We believe our three-strand triquetra approach offers a framework for the innovation required.

\section{Compliance with ethical standards}

Conflicts of interest None

\section{References}

1. Ayyala RS, Baird GL, Sze RW et al (2020) The growing issue of burnout in radiology - a survey-based evaluation of driving factors and potential impacts in pediatric radiologists. Pediatr Radiol 50:1071-1077 
2. Myers RA, Breen MA, Laor T (2020) A pandemic offers a silver lining for pediatric radiology training. Pediatr Radiol 50:15461550

3. Jamadar DA, Carlos R, Caoili EM et al (2005) Estimating the effects of informal radiology resident teaching on radiologist productivity: what is the cost of teaching? Acad Radiol 12:123-128

4. Davenport MS, Ellis JH, Khalatbari SH et al (2010) Effect of work hours, caseload, shift type, and experience on resident call performance. Acad Radiol 17:921-927

5. Glover M, Almeida RR, Schaefer PW et al (2017) Quantifying the impact of noninterpretive tasks on radiology report turn-around times. J Am Coll Radiol 14:1498-1503

6. Halabi SS, Prevedello LM, Kalpathy-Cramer J et al (2019) The RSNA pediatric bone age machine learning challenge. Radiology 290:498-503

7. Hayes SA, Breen M, McLaughlin PD et al (2014) Communication of unexpected and significant findings on chest radiographs with an automated PACS alert system. J Am Coll Radiol 11:791-795
8. Risko EF, Anderson N, Sarwal A et al (2012) Everyday attention: variation in mind wandering and memory in a lecture. Appl Cogn Psychol 26:234-242

9. Sajedi P, Salamon N, Hostetter J et al (2019) Reshaping radiology precall preparation: integrating a cloud-based PACS viewer into a flipped classroom model. Curr Probl Diagn Radiol 48:441-447

10. Tsai A, Barnewolt CE, Prabhu SP et al (2017) Creation and validation of a simulator for neonatal brain ultrasonography: a pilot study. Acad Radiol 24:76-83

11. Faulkner AR, Bourgeois AC, Bradley YC et al (2015) Simulationbased educational curriculum for fluoroscopically guided lumbar puncture improves operator confidence and reduces patient dose. Acad Radiol 22:668-673

Publisher's note Springer Nature remains neutral with regard to jurisdictional claims in published maps and institutional affiliations. 